Research Article

\title{
Effect of Hataedock Treatment on Epidermal Structure Maintenance through Intervention in the Endocannabinoid System
}

\author{
Hee-Yeon Kim, ${ }^{1,2}$ Sang-hyun Ahn, ${ }^{3}$ In-Jun Yang $\mathbb{D}^{1},{ }^{4}$ Sun-Young Park, ${ }^{5}$ \\ and Kibong Kim $\mathbb{1}^{1,2}$ \\ ${ }^{1}$ Department of Korean Pediatrics, Pusan National University Korean Medicine Hospital, Geumo-ro 20, Mulgeum-eup, \\ Yangsan-si, Gyeongsangnam-do 50612, Republic of Korea \\ ${ }^{2}$ Department of Korean Pediatrics, School of Korean Medicine, Pusan National University, Pusandaehak-ro 49, Mulgeum-eup, \\ Yangsan-si, Gyeongsangnam-do 50612, Republic of Korea \\ ${ }^{3}$ Department of Anatomy, College of Korean Medicine, Semyung University, Semyung-ro 65, Jecheon-si, \\ Chungcheongbuk-do 27136, Republic of Korea \\ ${ }^{4}$ Department of Physiology, College of Korean Medicine, Dongguk University, Dongdae-ro 123, Gyeongju-si, \\ Gyeongsangbuk-do 38066, Republic of Korea \\ ${ }^{5}$ Department of Physiology, College of Korean Medicine, Semyung University, Semyung-ro 65, Jecheon-si, \\ Chungcheongbuk-do 27136, Republic of Korea
}

Correspondence should be addressed to Kibong Kim; kkb2630@gmail.com

Received 1 October 2019; Revised 16 December 2019; Accepted 3 January 2020; Published 23 January 2020

Academic Editor: Mario Ledda

Copyright $(2020$ Hee-Yeon Kim et al. This is an open access article distributed under the Creative Commons Attribution License, which permits unrestricted use, distribution, and reproduction in any medium, provided the original work is properly cited.

The aim of this study was to investigate the efficacy of Hataedock (HTD) on skin barrier maintenance through the endocannabinoid system (ECS) intervention in Dermatophagoides farinae-induced atopic dermatitis (AD) NC/Nga mice. Douchi (fermented Glycine max Merr.) extracts prepared for HTD were orally administered to NC/Nga mice at a $20 \mathrm{mg} / \mathrm{kg}$ dose. Then, Dermatophagoides farinae extract (DfE) was applied to induce AD-like skin lesions during the 4th-6th and 8th-10th weeks. Changes in the epidermal structure of the mice were observed by histochemistry, immunohistochemistry, and TUNEL assay. The results showed that HTD significantly reduced the clinical scores $(p<0.01)$ and effectively alleviated the histological features. In the experimental groups, increased expression of cannabinoid receptor type (CB) 1, CB2, and G protein-coupled receptor 55 (GPR55) and distribution of filaggrin, involucrin, loricrin, and longevity assurance homolog 2 (Lass2) indicated that HTD maintained the epidermal barrier through intervening in the ECS. The expression of E-cadherin and glutathione peroxidase 4 (GPx4) was increased, and the levels of cluster of differentiation 1a (CD1A) were low. Moreover, the apoptosis of inflammatory cells was elevated. The production of phosphorylated extracellular signal-related kinase (pERK), phosphorylated c-Jun amino-terminal kinase (p-JNK), and phosphorylated mammalian target of rapamycin (p-mTOR) was low, and epidermal thickness was decreased. Besides, the expression levels of involucrin were measured by treating genistein, an active ingredient of Douchi extract, and palmitoylethanolamide (PEA), one of the ECS agonists. The results showed that genistein had a better lipid barrier formation effect than PEA. In conclusion, HTD alleviates the symptoms of AD by maintaining skin homeostasis, improving skin barrier formation, and downregulating inflammation, through ECS intervention.

\section{Introduction}

Atopic dermatitis (AD) is an inflammatory skin disease, highly relapsing, characterized by prorates, dryness, and erythematous eczema [1] and is also the initial stage of an atopic march that progresses to asthma and allergic rhinitis [2]. The pathogenesis of $\mathrm{AD}$ has not yet been elucidated and is thought to be caused by a combination of genetic, immunological, and environmental factors and skin barrier dysfunction [3]. Among them, skin barrier dysfunction has become the most important factor since the outside-inside hypothesis in the 1990s that damage to 
the skin barrier was the early mechanism of $\mathrm{AD}$ pathogenesis [4].

Levels of various keratinocyte differentiation markers, including filaggrin, involucrin, and loricrin, are low in AD lesions [5]. Moreover, levels of ceramide in stratum corneum (SC) are reduced [6]. Hallmarks of AD skin are epidermal hyperplasia resulting from increased epidermal proliferation and reduced differentiation [7] and spongiosis resulting from tissue remodeling [8]. These structural changes in the skin barrier disrupt skin homeostasis, preventing the skin from performing its normal barrier function.

The endocannabinoid system (ECS) is a biological system composed of cannabinoids (CBs) that regulate appetite, pain sensation, mood, and memory [9]. Recently, it was revealed that an increase or decrease of the ECS tones is associated with the various pathological conditions [10]. Temporarily altered activity of ECS reduces the symptoms of the body's compensatory response or slows disease progression. In other cases, activation of ECS can act as a pathogenic or reflect a defect in the body [11]. In particular, the ECS is associated with the regulation of cell growth, proliferation, immunity, and the inflammatory response involved in skin homeostasis [12]. Representative CBs, anandamide ( $\mathrm{N}$-arachidonoylethanolamine; AEA) and 2-arachidonoylglycerol (2-AG), which are produced locally in various cellular compartments of the skin, regulate the various cutaneous functions via binding to cannabinoid receptor type (CB)1 or CB2 [13]. In epidermal keratinocytes, activation of $\mathrm{CB} 1$ and $\mathrm{CB} 2$ suppresses cellular proliferation and differentiation [14], releases the inflammatory mediators [15], and induces apoptosis [16]. Additionally, $\mathrm{CB} 1$ suppresses the secretion of proinflammatory chemokines to help control skin inflammation [17]. In the hair follicle, activation of CB1 attenuates hair growth and proliferation, whereas promotes apoptosis and the regression phase [18]. In the sebaceous gland, activation of CB2 stimulates lipid formation and apoptosis [19]. Furthermore, various CBs inhibit sensory phenomena such as pain and itching via CB1 $[20,21]$. ECS constitutively regulates the well-balanced proliferation and differentiation of skin cells, as well as immune and inflammatory responses. The fine-tuned changes in ECS might promote or alleviate skin diseases [13]. Therefore, the ECS is a microenvironmental control factor for maintaining skin homeostasis. Thus, the role of the ECS as a new therapeutic target for skin diseases has been highlighted [22].

In traditional Chinese medicine, inflammatory diseases such as asthma, rhinitis, and $\mathrm{AD}$ are thought to be due to heat syndrome [23]. Therefore, inflammatory diseases are treated with heat-clearing herbal medicine to remove the accumulated heat in the body [24]. Fetal heat is caused by changes in the microimmune environment that affect fetal survival, such as in Th2-skewed conditions. Thus, fetal heat manifests as a variety of diseases in newborns by disrupting the homeostasis of the fetus, of which $\mathrm{AD}$ is the most common [25]. In Korean medicine, Hataedock (HTD), herbal extracts that are orally administered to neonates and infants, was used to clear fetal heat to prevent inflammatory diseases. Our previous studies have shown that HTD mitigates $\mathrm{AD}$ development due to fetal heat and controls Th2skewed conditions [26, 27]. Moreover, our studies showed that lipid barrier formation in the epidermis is increased after the application of HTD [28].

Douchi (fermented Glycine max Merr.), one of the most commonly used herbs in HTD, is a kind of fermented soybean known as a herb that reduces heat by radiating the body heat [29]. Recently, many studies have been conducted on ECS modulators, especially soybean [30-32]. In particular, genistein, a modest fatty acid amide hydrolase (FAAH) inhibitor, can prevent proinflammatory response in a CB1/ CB2-dependent manner [33]. In our previous study, we found that Douchi extract contains more genistein than raw soybeans [26].

HTD is a prophylactic treatment applied to neonates and infants before an inflammatory disease occurs and is not a treatment of inflammatory disease. However, there have been no studies on the mechanism by which HTD can prevent inflammatory diseases. Therefore, based on the results of inflammation relief and lipid barrier formation induced by HTD, we hypothesized that HTD intervenes in the ECS to maintain skin homeostasis and that the ECS maintains the epidermal structure by influencing microenvironmental control of the epidermis. Here, we investigated changes in the epidermal structure to confirm how HTD influences AD alleviation through ECS intervention.

\section{Materials and Methods}

2.1. Preparation of HTD Herbal Extracts. In this study, the Douchi (豆鼓, fermented Glycine max Merr.) was purchased from Namyoung Pharm (Muju, Republic of Korea). Douchi is a black bean fermented for 5 days at $37-38^{\circ} \mathrm{C}$ in Artemisia Apiacea Herba and Mori Folium extract $(1: 1)$. The Douchi extract was prepared as follows: (1) $100 \mathrm{~g}$ of Douchi was boiled for 3 hours in $1 \mathrm{~L}$ of distilled water and then filtered, and (2) the filtrate was concentrated to $50 \mathrm{~mL}$ by using a rotatory vacuum evaporator and then freeze-dried to obtain $15 \mathrm{~g}$ of the extract (yield: 15.0\%).

2.2. Experimental Animals and $A D$ Induction. In this study, we used 3-week-old male NC/Nga mice (14.3 $\pm 0.3 \mathrm{~g}$ each) purchased from Central Lab Animal Inc. (Seoul, Republic of Korea). The animals were kept under standard laboratory conditions $\left(25^{\circ} \mathrm{C}\right.$ and $12 / 12 \mathrm{~h}$ light/dark cycles) and standard food and water were freely provided. The mice were allocated randomly to 3 groups ( $n=10$ per group) as follows: the normal group (Ctrl group), AD-induced with no treatment group (AE group), and AD-induced with HTD treatment group (FGT group). In the FGT group, 3-week-old male NC/ Nga mice were orally administered Douchi extracts at $20 \mathrm{mg} / \mathrm{kg}$ on the $1^{\text {st }}, 2^{\text {nd }}$, and $3^{\text {rd }}$ days were used for HTD treatment. To induce AD-like skin lesions, the back skin regions of the mice were exposed and $1 \mathrm{~mL}$ of $5 \%$ sodium dodecyl sulfate (Sigma-Aldrich, St. Louis, MO, USA) was rubbed 20 times to remove the lipid lamella of the SC of each mouse. Then, Dermatophagoides farinae extract (DfE) (100 mg, Biostir Inc., Osaka, Japan) was administered at the $4^{\text {th }}, 5^{\text {th }}$, and $6^{\text {th }}$ weeks and administered again at the $8^{\text {th }}, 9^{\text {th }}$, and $10^{\text {th }}$ weeks. In the $11^{\text {th }}$ week, the mice were sacrificed 
with sodium pentobarbital. All animal experiments were approved by the Institutional Animal Care and Use Committee of Pusan National University (IACUC number: PNU2015-0924). We followed the National Institutes of Health (NIH) Guide for the Care and Use of Laboratory Animals throughout this study. The overall experimental protocol is summarized in Figure 1.

2.3. Dermatitis-Induced Animal Model. To induce skin lesions, the back skin regions of the mice were exposed and $1 \mathrm{~mL}$ of $5 \%$ sodium dodecyl sulfate (Sigma-Aldrich, St. Louis, MO, USA) was rubbed 20 times to remove the lipid lamella of the SC of each mouse. The mice were allocated randomly to 4 groups ( $n=10$ per group) as follows: the normal group (Ctrl group), dermatitis-induced with no treatment group (DE group), dermatitis-induced with genistein $10 \mathrm{mg} / \mathrm{kg}$ treatment group (GT group), and dermatitis-induced with palmitoylethanolamide (PEA) $10 \mathrm{mg} /$ $\mathrm{kg}$ treatment group (PT group). In the GT and PT groups, 6week-old male BALB/c mice were orally administered genistein and PEA for 3 days.

2.4. Western Blotting. 3D human skin keratinocytes were dispensed into the 6 -well plate as $4 \times 105$ cells/well, cultured in $80 \%$ confluence, and then cultured for 24 hours after treated with reagents. After culture, the lysate was prepared by treating with RIPA buffer (Atto, Tokyo, Japan) and centrifuged at $12,000 \mathrm{rpm}$ at $4^{\circ} \mathrm{C}$ for 10 minutes to recover the supernatant. After protein quantification was performed using the Bradford protein assay reagent, $50 \mu \mathrm{g}$ of proteins was separated by SDSpolyacrylamide gel $(10 \%)$ and transferred to a polyvinylidene difluoride (PVDF) membrane at $190 \mathrm{~mA}$ for 80 minutes. The membrane was blocked in PBS containing 5\% skim milk for 1 hour at $37^{\circ} \mathrm{C}$ and treated overnight with an anti-involucrin antibody (\# ab24722, \# ab53112, Cambridge, MA, USA). In addition, it was treated with HRP-conjugated anti-rabbit antibody at room temperature for 1 hour. After completion of the reaction, the membrane was developed using an enhanced chemiluminescence system (Bio-Rad Laboratories, Hercules, USA) and observed for protein expression on X-ray film.

Skin tissues were homogenized with ice-cold tissue extraction reagent (Thermo Fisher Scientific, Vienna, Austria) containing protease and phosphatase inhibitors (Atto, Tokyo, Japan) and centrifuged at 10,000 rpm for 20 minutes, and supernatants were collected. Amounts of proteins in samples were determined using Bradford protein assay reagent (Bio-Rad, Hercules, CA, USA). Proteins $(50 \mu \mathrm{g})$ were separated by $10 \%$ SDS-PAGE electrophoresis and transferred to polyvinylidene difluoride membranes (Merck Millipore, Carrigtwohill, Ireland), which were blocked with 5\% skim milk in PBS for 2 hours at room temperature, and incubated with primary antibodies, followed by secondary antibody horseradish peroxidase-conjugated anti-IgG. Anti-involucrin was purchased from Santa Cruz Biotech (Paso Robles, CA, USA). All bands were detected by enhanced chemiluminescence (Bio-Rad, Hercules, CA, USA).
2.5. Evaluation of Clinical Symptoms. The morphologic severity of the dorsal skin was evaluated after 3 weeks and compared to the baseline. The evaluation items of the skin score are as follows: (1) erythema/hemorrhage, (2) scarring/ dryness, (3) edema, and (4) excoriation/erosion were scored as 0 (none), 1 (mild), 2 (moderate), or 3 (severe). The sum of the individual scores was defined as the AD skin score [34]. Transepidermal water loss (TEWL) was measured for 30 seconds using a Vapometer device (Delfin Technologies, Finland) in accordance with the manufacturer's manual.

2.6. Angiogram. The capillary distribution was photographed at $4 \mathrm{x}$ magnification using a sharpen low-filter in Image-Pro Plus (Media Cybernetics, Rockville, USA) and then inverted (180-200 intensity range).

2.7. Tissue Processing and Histochemistry. After sacrificing the mice, the obtained dorsal skin was fixed with $10 \% \mathrm{NBF}$ at room temperature for 24 hours, and the fixed skin tissues were embedded in paraffin. Then, skin samples at a thickness of $5 \mu \mathrm{m}$ were histochemically stained. To examine histological changes such as collagen fiber distribution and epithelial hyperplasia, we performed Masson's trichrome (M/ T) staining. Toluidine blue staining was performed to investigate the distribution and morphological changes in the intercellular space of keratinocytes. To investigate the inflammatory changes in the epidermis, we performed phloxine-tartrazine $(\mathrm{P} / \mathrm{T})$ staining.

2.8. Immunohistochemistry. The skin samples were treated with proteinase $\mathrm{K}$ solution $(20 \mu \mathrm{g} / \mathrm{mL})$ for 5 minutes at room temperature, and the proteolyzed skin samples were incubated in blocking serum ( $10 \%$ normal goat serum) for 4 hours. Then, the skin samples were incubated with primary antibodies (all antibodies used in the experiment were purchased from Santa Cruz Biotechnology, Dallas, TX, USA), including goat anti-interleukin- (IL-) 4 (IL-4; $1: 100$ ), goat anti-CB1 $(1: 100)$, goat anti-CB2 $(1: 100)$, goat anti-G protein-coupled receptor 55 (GPR55; $1: 100$ ), rabbit antifilaggrin $(1: 100)$, rabbit anti-involucrin $(1: 50)$, rabbit antiloricrin $(1: 50)$, goat anti-longevity assurance homolog 2 (Lass2; $1: 100)$, goat anti-protein kinase C (PKC; $1: 100)$, goat anti-E-cadherin $(1: 100)$, goat anti-glutathione peroxidase $4(\mathrm{GPx} 4 ; 1: 100)$, goat anti-cluster of differentiation 1a (CD1A; 1:100), goat anti-phosphorylated extracellular signal-related kinase (p-ERK; $1: 100$ ), goat anti-phosphorylated c-Jun amino-terminal kinase (p-JNK; $1: 100)$, and goat anti-phosphorylated mammalian target of rapamycin (p-mTOR; $1: 100$ ) for 72 hours in a $4^{\circ} \mathrm{C}$ humidified chamber. Next, the samples were incubated with biotinylated rabbit anti-goat $\operatorname{IgG}(1: 100)$, secondary antibody, at room temperature for 24 hours. Then, an avidin-biotin complex kit (Vector Lab, Burlingame, CA, USA) was used at room temperature for 1 hour. As a final step, the samples were treated with $0.05 \mathrm{M}$ Tris- $\mathrm{HCl}$ buffer solution $(\mathrm{pH} 7.4)$ composed of $0.05 \% 3,3^{\prime}$-diaminobenzidine and $0.01 \% \mathrm{HCl}$ and counterstained with hematoxylin. 


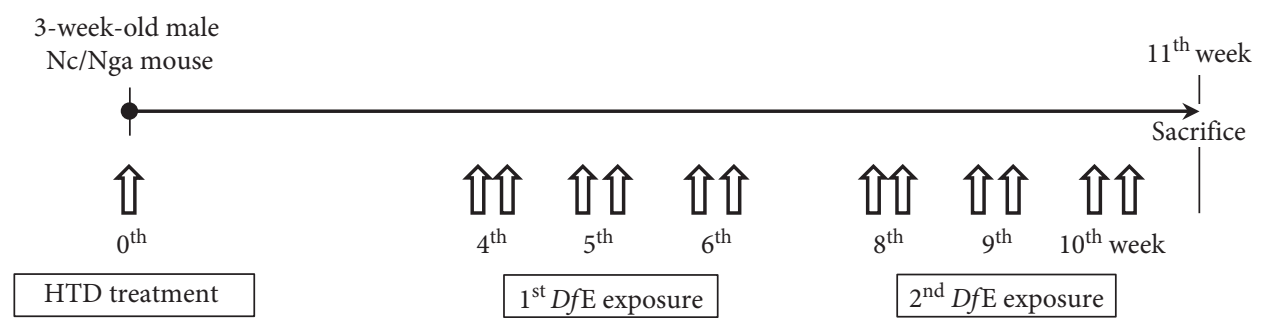

Figure 1: Experiment design. Prior to inducing AD-like skin lesion, FGT group was applied with Douchi (fermented Glycine max Merr.) extracts orally on days 1,2 , and 3 . The mice were repeatedly induced by DfE at the $4^{\text {th }}, 5^{\text {th }}, 6^{\text {th }}, 8^{\text {th }}, 9^{\text {th }}$, and $10^{\text {th }}$ weeks. HTD: Hataedock and DfE: Dermatophagoides farinae extract.

2.9. TUNEL Assay. The terminal deoxynucleotidyl transferase-mediated dUTP-digoxigenin nick end labeling (TUNEL) assay was conducted using an in situ apoptosis detection kit (Apoptag, Intergen, New York, USA) to observe apoptosis. The skin samples were incubated with proteinase K solution $(20 \mu \mathrm{g} / \mathrm{mL})$ for 5 minutes and treated with equilibration buffer solution for 5 seconds. Then, the samples were reacted with TdTenzyme $(36 \mu \mathrm{L}$ TdT enzyme: $72 \mu \mathrm{L}$ reaction buffer). Subsequently, the samples were incubated in a humidified chamber at $37^{\circ} \mathrm{C}$ for 1 hour and washed in the intensity stop/wash buffer for 10 minutes, and the reaction was terminated with anti-digoxigenin peroxidase and DAB for 1 hour. We then observed the counterstained sections with eosin using an optical microscope.

2.10. Image Analysis and Statistical Analysis. The results of the immunohistochemical and TUNEL assays were digitized by image analysis using Image-Pro Plus (Media Cybernetics). The data are presented as the mean \pm standard error. The image analysis was performed at $400 \mathrm{x}$ magnification of randomly selected fields of each group. The statistical data were analyzed with SPSS software (SPSS 23, SPSS Inc., Chicago, IL, USA). The significance was verified by using a one-way analysis of variance (ANOVA) and Levene's (LSD) test with a significance level of $p<0.01$.

\section{Results}

3.1. Alleviation of $A D$. HTD-mediated alleviation of $\mathrm{AD}$ was estimated based on external morphology, clinical symptoms, AD skin scores, and angiogenesis. The external image of the AE group showed the most severe pathological features such as erythema, hemorrhage, scarring, dryness, edema, erosion, and excoriation. In contrast, HTD alleviated AD symptoms in the FGT group (Figure 2). The AD skin scores were evaluated at 7.2 \pm 0.25 in the AE group and $3.9 \pm 0.28$ in the FGT group. Significant differences were found as the skin scores of the FGT group were decreased by $46 \%(p<0.01)$ compared with those of the AE group (Figure 2).

To compare AD-associated angiogenesis, we conducted angiograms in the AE and FGT groups. The angiogenesis was $59,153 \pm 996 / 20,000,000$ pixels in the AE group, which was $271 \%(p<0.01)$ higher than that of the Ctrl group. In contrast, the angiogenesis in the FGT group was $36,705 \pm 861 / 20,000,000$ pixels which were decreased by $38 \%$ $(p<0.01)$ compared with that of the $\mathrm{AE}$ group. The angiogram indicated that angiogenesis was more suppressed in the FGT group than in the AE group (Figure 2).

In addition, we used $\mathrm{M} / \mathrm{T}$ staining to observe papillary dermal edema. The results showed that papillary dermal edema and capillary distribution were increased and that the distribution of collagen fibers in the dermis was decreased in the $\mathrm{AE}$ group. Moreover, the infiltration of inflammatory cells into the basement of the epidermis in the AE group was significantly increased. In contrast, papillary dermal edema was significantly decreased in the HTD treatment group (Figure 2).

One of the important features of $\mathrm{AD}$, the expression of increased Th2 cytokines, was estimated by measuring IL-4positive reactions in the cytoplasm of papillary dermal cells. IL-4-positive reactions in the AE group were increased by $345 \%(p<0.01)$ compared with those of the Ctrl group. In contrast, the IL-4-positive reactions in the FGT group were decreased by $34 \%(p<0.01)$ compared with those of the AE group. Significant differences were shown between all of the groups $(p<0.01)$ (Figure 2$)$.

Overall, these results suggest that HTD alleviates the development of AD in NC/Nga mice.

3.2. Activation of the ECS. Recent studies revealed that the ECS is involved in the regulation of skin homeostasis by controlling cell growth, differentiation, and immune and inflammatory responses [13]. Thus, the inadequate function of the ECS is directly related to skin disorders.

To confirm the activation of the ECS, we measured the expression levels of CB1, CB2, and GPR55 through immunohistochemical staining. Compared with the levels in the AE group, HTD significantly increased the expression levels of CB1, CB2, and GPR55. The levels of CB1 expression in the FGT group were increased by $476 \% \quad(p<0.01)$ compared with those of the AE group. The FGT group showed a $121 \%(p<0.01)$ increase in CB2 expression compared with that of the AE group. In addition, the levels of GPR55 expression in the FGT group were increased by $147 \%(p<0.01)$ compared to those in the AE group (Figure 3). These results suggest that HTD has the potential to suppress skin disorders through the intervention of ECS.

3.3. Regulation of the Lipid Barrier. The cornified cell envelope (CE), which is composed of filaggrin, involucrin, loricrin, and other proteins, is important in the skin barrier [35]. Moreover, recent studies revealed that reduced 


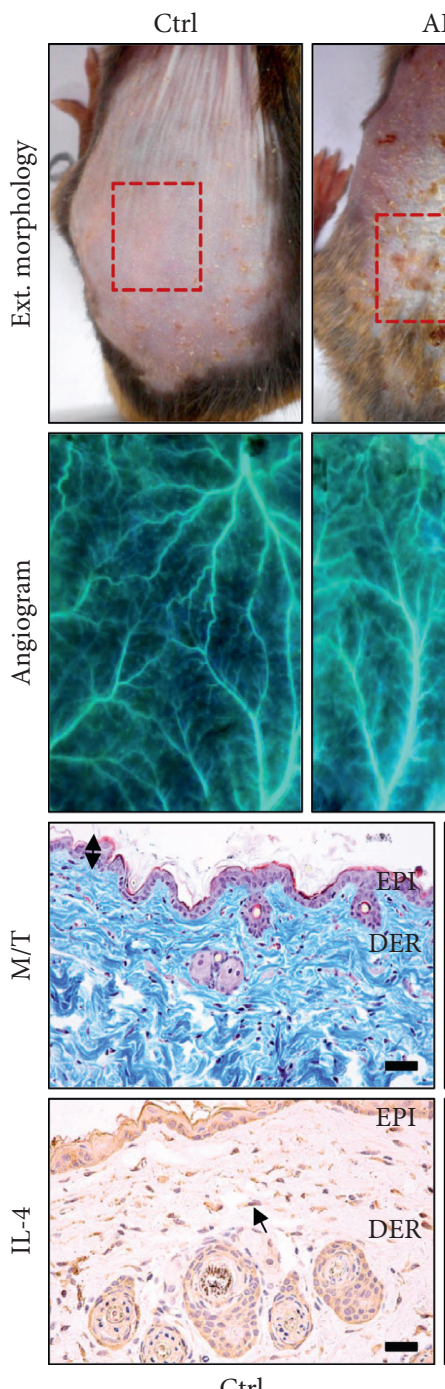

$\mathrm{AE}$
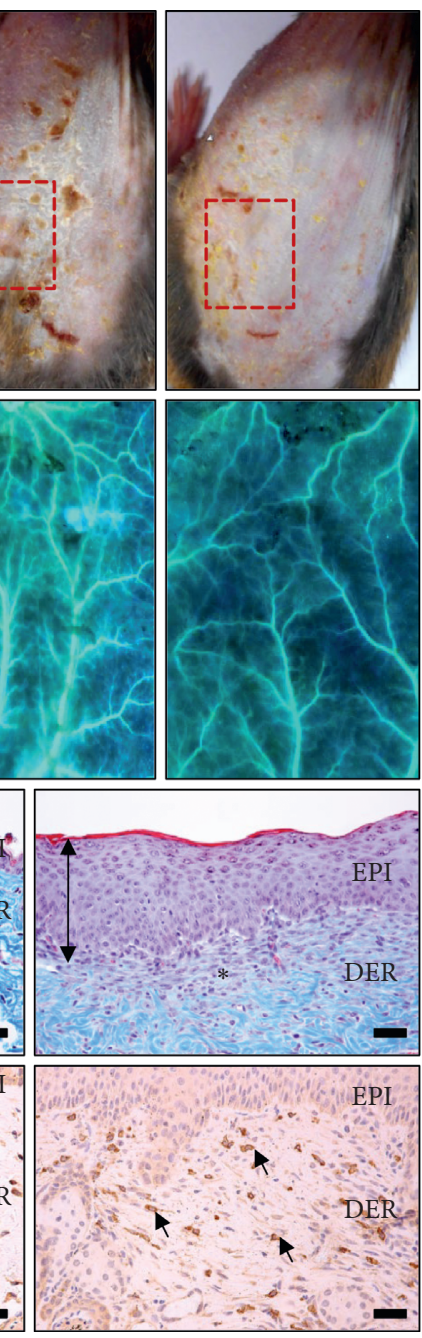

$\mathrm{AE}$

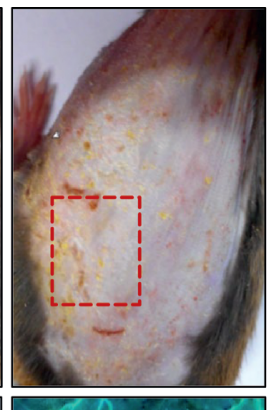

FGT
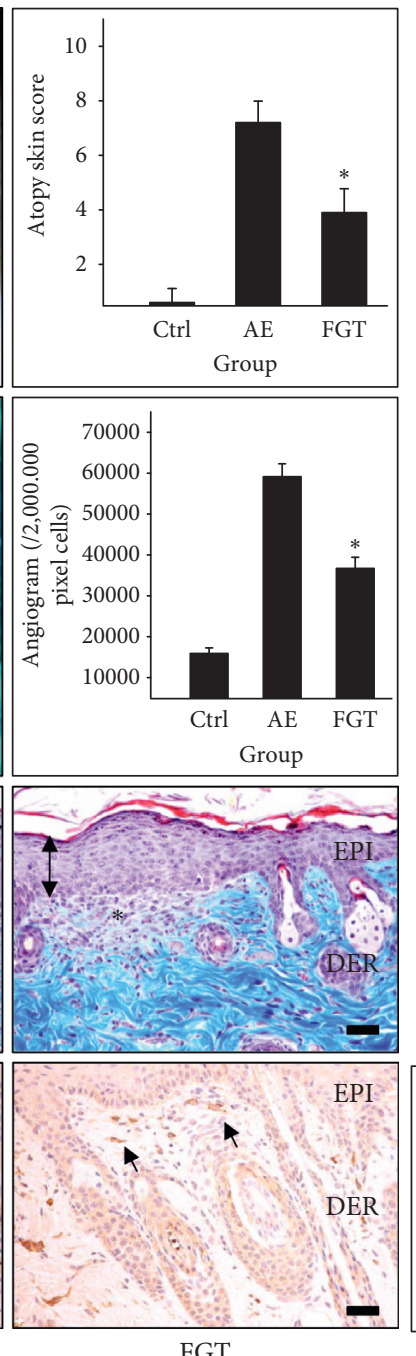

FGT

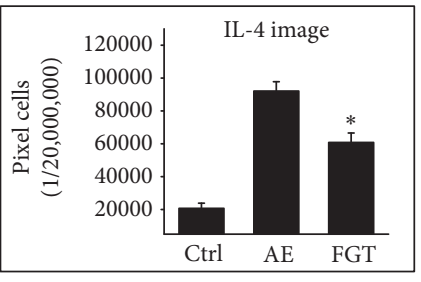

FIgURE 2: The alleviation effects on symptoms of AD. HTD relieved the AD-like skin lesions in the FGT group. The capillary distribution is increased in the AE group but decreased in the FGT group (4x) $(p<0.01)$. In M/T staining result, edema of papillary dermis is decreased in the FGT group, but increased in the AE group $(p<0.01)(\mathrm{M} / \mathrm{T}$ staining; asterisk, edema invoked region; bar size, $50 \mu \mathrm{m})$. The IL-4-positive reactions (arrow indicates dark brown) were decreased in the AE group compared with the FGT group $(p<0.01)$ (immunohistochemistry; bar size, $50 \mu \mathrm{m}) .{ }^{*} p<0.01$, compared with the AE group. Ctrl: normal, AE: AD-induced with no treatment, FGT: AD-induced with HTD treatment, EPI: epidermis, DER: dermis, and M/T: Masson's trichrome staining.

expression of involucrin and loricrin in $\mathrm{AD}$ could exacerbate $\mathrm{AD}[36]$. These studies indicated that an abnormal epidermal barrier is not simply an epiphenomenon of AD but rather promotes inflammation in $\mathrm{AD}$ [37].

To determine the effect of HTD on the epidermal lipid barrier, we measured the levels of filaggrin, involucrin, and loricrin and the levels of PKC and Lass2, which are involved in epidermal lipid metabolism [38, 39].

The levels of filaggrin in keratohyalin granules in the SC were markedly reduced in the $\mathrm{AE}$ group, whereas the levels of filaggrin in the FGT group were increased by $91 \%$ $(p<0.01)$ compared with those in the AE group. Increased levels of involucrin were observed in the cornified layer of the SC in the FGT group. The levels of involucrin in the FGT group were increased by $210 \%(p<0.01)$ compared with those of the AE group.
Moreover, the levels of loricrin in the AE group were decreased by $74 \%(p<0.01)$ compared with those of the Ctrl group. This decrease was not observed in the FGT group. The levels of loricrin in the FGT group were increased by $170 \%$ $(p<0.01)$ compared with those of the AE group (Figure 4).

Levels of Lass2, a ceramide synthase, were $29,821 \pm 627$ / $20,000,000$ pixels which were markedly decreased by $69 \%$ $(p<0.01)$ compared with those of the Ctrl group. However, HTD increased the levels of Lass 2 in the FGT group by $142 \%$ $(p<0.01)$ compared with those of the AE group. The levels of Lass2 were 72,258 $\pm 1315 / 20,000,000$ pixels in the FGT group (Figure 4).

In the $\mathrm{AE}$ group, an increase in PKC-positive reactions was observed in damaged keratinocytes. However, this increase was not observed in the FGT group that was orally administered Douchi. The levels of PKC in the AE group 

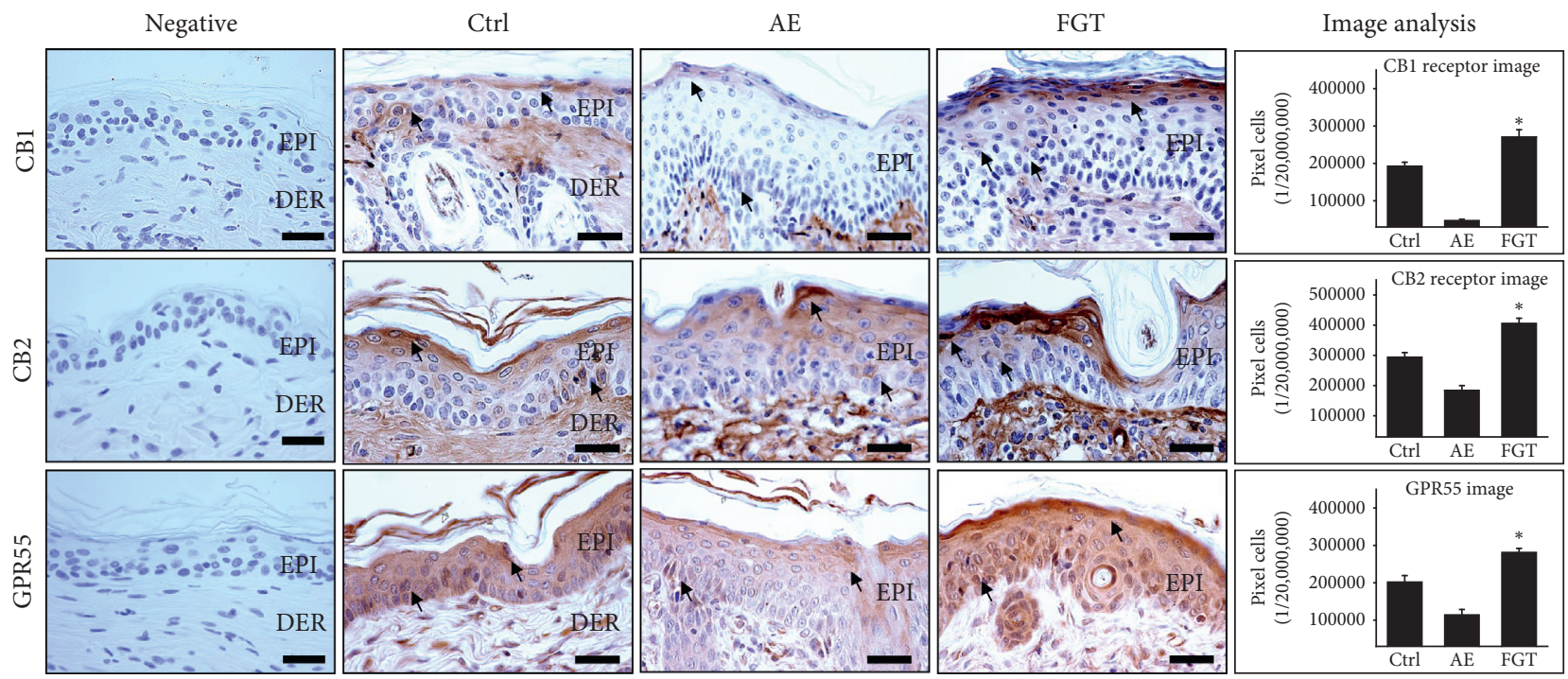

FIgURE 3: The ECS activation effects of HTD. HTD generates the ECS in the FGT group. The CB1, CB2, and GPR55 expression (arrow indicates dark brown) was significantly increased in the FGT group as compared with the AE group $(p<0.01)($ bar size, $50 \mu \mathrm{m})$. The data of $\mathrm{CB} 1, \mathrm{CB} 2$, and GPR55 image analysis showed the same results $(p<0.01) .{ }^{*} p<0.01$, compared with the AE group. Ctrl: normal, AE: ADinduced with no treatment, FGT: AD-induced with HTD treatment, EPI: epidermis, and DER: dermis.
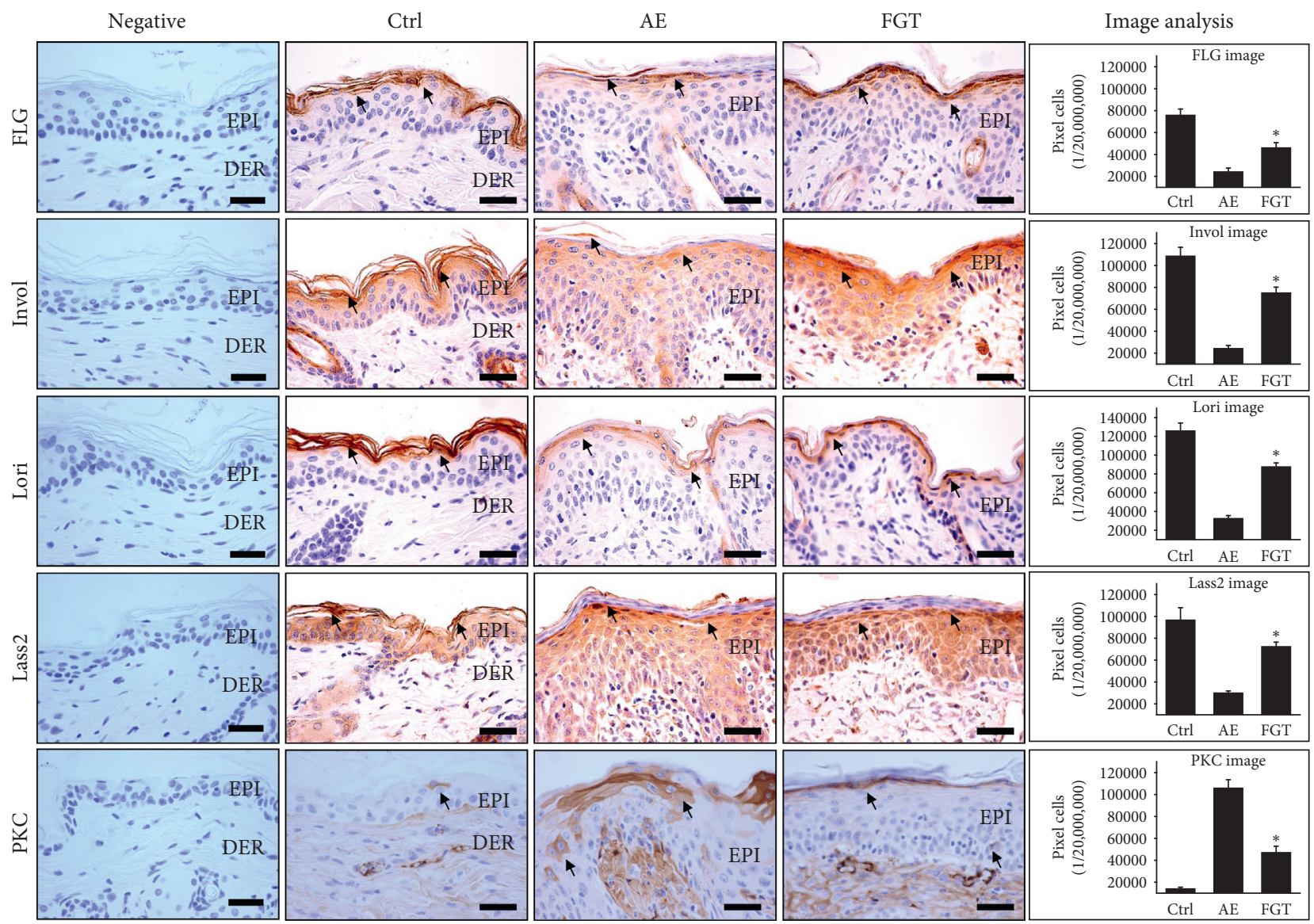

FIGURE 4: The lipid barrier regulatory effects of HTD. HTD repaired the lipid barrier in the FGT group. The filaggrin, involucrin, loricrin, and Lass2-positive reaction (arrow indicates dark brown) were significantly increased in the FGT group as compared with the AE group $(p<0.01)$ (bar size, $50 \mu \mathrm{m})$. The PKC-positive reaction (arrow indicates dark brown) was remarkably reduced in the FGT group compared with the AE group $(p<0.01)$ (bar size, $50 \mu \mathrm{m}) .{ }^{*} p<0.01$, compared with the AE group. Ctrl: normal, AE: AD-induced with no treatment, FGT: AD-induced with HTD treatment, EPI: epidermis, DER: dermis, FLG: filaggrin, Invol: involucrin, and Lori: loricrin. 
were $105,789 \pm 2451 / 20,000,000$ pixels which were significantly increased by $664 \%(p<0.01)$ compared with those of the Ctrl group. In the FGT group, the levels of PKC were $46,958 \pm 1874 / 20,000,000$ pixels which were significantly reduced by $56 \%(p<0.01)$ compared with those of the AE group (Figure 4 ).

Thus, by modulating filaggrin, involucrin, loricrin, Lass2, and PKC expression, HTD might serve as a highly effective agent for improving lipid barrier formation.

3.4. Regulation of Epidermal Structure. Typically, epidermal thickness due to epidermal hyperplasia and spongiosis is observed in $\mathrm{AD}$ skin lesions [40]. The spongiosis, which expands the intercellular spaces in the epidermal basal lamina, is formed by the impairment of intercellular adhesions between the epidermal keratinocytes [41]. We performed a comparison of epidermal thickness between the $\mathrm{AE}$ and FGT groups through toluidine blue staining. The results showed that the intercellular spaces of the $\mathrm{AE}$ group were significantly increased compared to those of the Ctrl group. On the other hand, HTD reduced the intercellular spaces to a level comparable to that in the Ctrl group. In addition, the migration of lymphocytes and the distribution of capillaries in the dermal papilla of the AE group were significantly increased compared with those in the Ctrl group. In contrast, the extent of lymphocyte migration and distribution of capillaries were significantly decreased in the HTD treatment group (Figure 5). These findings indicate that the expansion of intercellular space causes the basement membrane to collapse and lymphocytes to migrate.

To examine the effect of HTD on the cell-cell junctions of the epidermal structure, we measured the levels of E-cadherin, which is an adherens junction protein. E-cadherin expression was markedly reduced in the $\mathrm{AE}$ group, whereas the levels of E-cadherin in the FGT group were increased by $347 \%(p<0.01)$ as compared with those of the AE group. The levels of E-cadherin in the AE group were 12,552 \pm 722 / $20,000,000$ pixels which were markedly decreased by $68 \%$ $(p<0.01)$ compared with those of the Ctrl group. However, the levels of E-cadherin in the FGT group were 56,147 \pm 1559 / $20,000,000$ pixels, which were higher than those of the Ctrl group (Figure 5).

Recent, studies revealed that the expression of GPx4, an antioxidant enzyme, is increased by the induction of transcriptional response to oxidative stress in the skin of $\mathrm{AD}$ patients [42]. Moreover, GPx4 regulates apoptosis by releasing the apoptogenic proteins [43]. Thus, we examined the levels of GPx4 to investigate the protective effect of HTD against oxidative damage by oxidative stress. The levels of GPx4 in the AE group were 40,451 $\pm 798 / 20,000,000$ pixels which were markedly increased by $235 \%(p<0.01)$ compared with those of the Ctrl group. The expression of GPx4 in the FGT group was $61,253 \pm 987 / 20,000,000$ pixels, which was $51 \%(p<0.01)$ higher than that of the AE group (Figure 5). These findings mean that HTD acts as a defense against oxidative stress by increasing the expression of GPx4 and contributes to maintaining cell homeostasis through the regulation of apoptosis.
CD1A is often used as a marker for evaluating the number of epidermal Langerhans cells [44]. Recent studies have reported that $\mathrm{CD} 1 \mathrm{~A}$ expression is increased in $\mathrm{AD}$ skin lesions compared to that of nonlesions [45]. Moreover, the disruption of the epidermal structure allows skin resident antigen-presenting cells such as Langerhans cells to capture environmental antigens [46]. Therefore, we tried to confirm the destruction of the epidermal structure through CD1A staining. In the $\mathrm{AE}$ group, an increase in $\mathrm{CD} 1 \mathrm{~A}$ expression was observed. The levels of $\mathrm{CD} 1 \mathrm{~A}$ in the $\mathrm{AE}$ group were $78,693 \pm 1506 / 20,000,000$ pixels which were significantly increased by $661 \%(p<0.01)$ compared with those of the Ctrl group. On the other hand, the levels of CD1A in the FGT group were $32,167 \pm 811 / 20,000,000$ pixels which were significantly reduced by $59 \%(p<0.01)$ compared with those of the AE group (Figure 5).

Furthermore, the TUNEL assay demonstrated that the HTD maintains the epidermal structure by promoting apoptosis between the stratum spinosum (SP) and stratum basale (SB). In the AE group, the number of apoptotic cells was $30,360 \pm 682 / 20,000,000$ pixels which were increased by $150 \%$ compared with that of the Ctrl group. However, the number of apoptotic cells in the FGT group was $50,373 \pm 1119 / 20,000,000$ pixels which were markedly increased by $66 \%(p<0.01)$ in contrast to the increase in the AE group (Figure 5). These results indicate that HTD maintains the epidermal structure by inducing the apoptosis of inflammatory cells.

Thus, by modulating the intercellular space, expression of E-cadherin, GPx4, and CD1A, and apoptosis, HTD might serve as a highly effective therapy for improving epidermal structures.

3.5. Regulation of Epidermal Inflammation. To estimate the anti-inflammatory effects of $\mathrm{HTD}$, we performed $\mathrm{P} / \mathrm{T}$ staining and measured the expression levels of p-ERK, $\mathrm{p}$-JNK, and p-mTOR.

The results of $\mathrm{P} / \mathrm{T}$ staining showed that the infiltration of inflammatory cells such as granulocytes and lymphocytes in the $\mathrm{AE}$ group was significantly increased compared to that of the Ctrl group. On the other hand, the infiltration of inflammatory cells after HTD was reduced to a level similar to that of the Ctrl group (Figure 6).

In the cell, inflammatory signals activate various signaling molecules, among which mitogen-activated protein kinases (MAPKs) are key signaling molecules that activate various transcription factors through phosphorylation [47]. MAPKs, including ERK and JNK, are involved in the activation of transcription factors including nuclear factor kappa-light-chain-enhancer of activated B cells (NF- $\kappa B$ ) and activator protein 1 (AP-1), thereby increasing the secretion of inflammatory mediators [48]. Thus, the levels of p-ERK and p-JNK were evaluated to investigate the effect of HTD on the activation of phosphorylated MAPKs. The levels of p-ERK in the AE group were 80,166 $\pm 1399 / 20,000,000$ pixels which were significantly increased by $665 \% \quad(p<0.01)$ compared with those of the Ctrl group. In contrast, the levels of p-ERK in the FGT group were $34,803 \pm 1001 / 20,000,000$ 


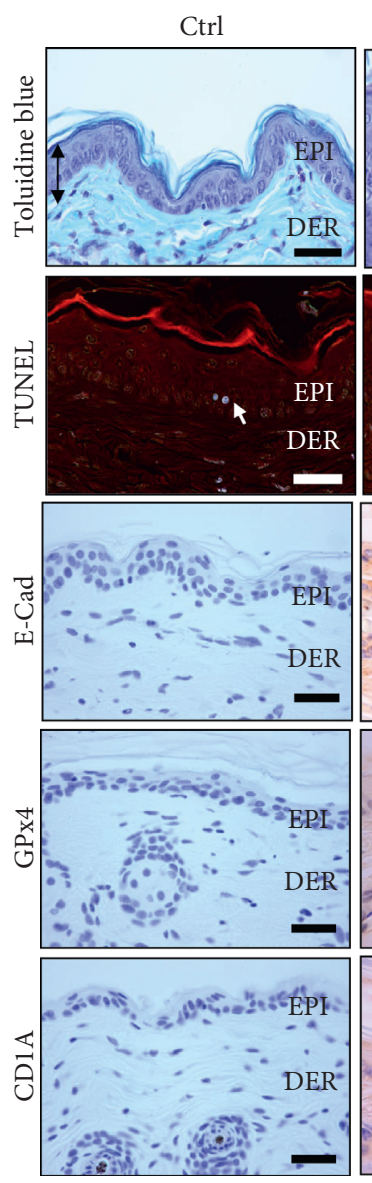

Negative
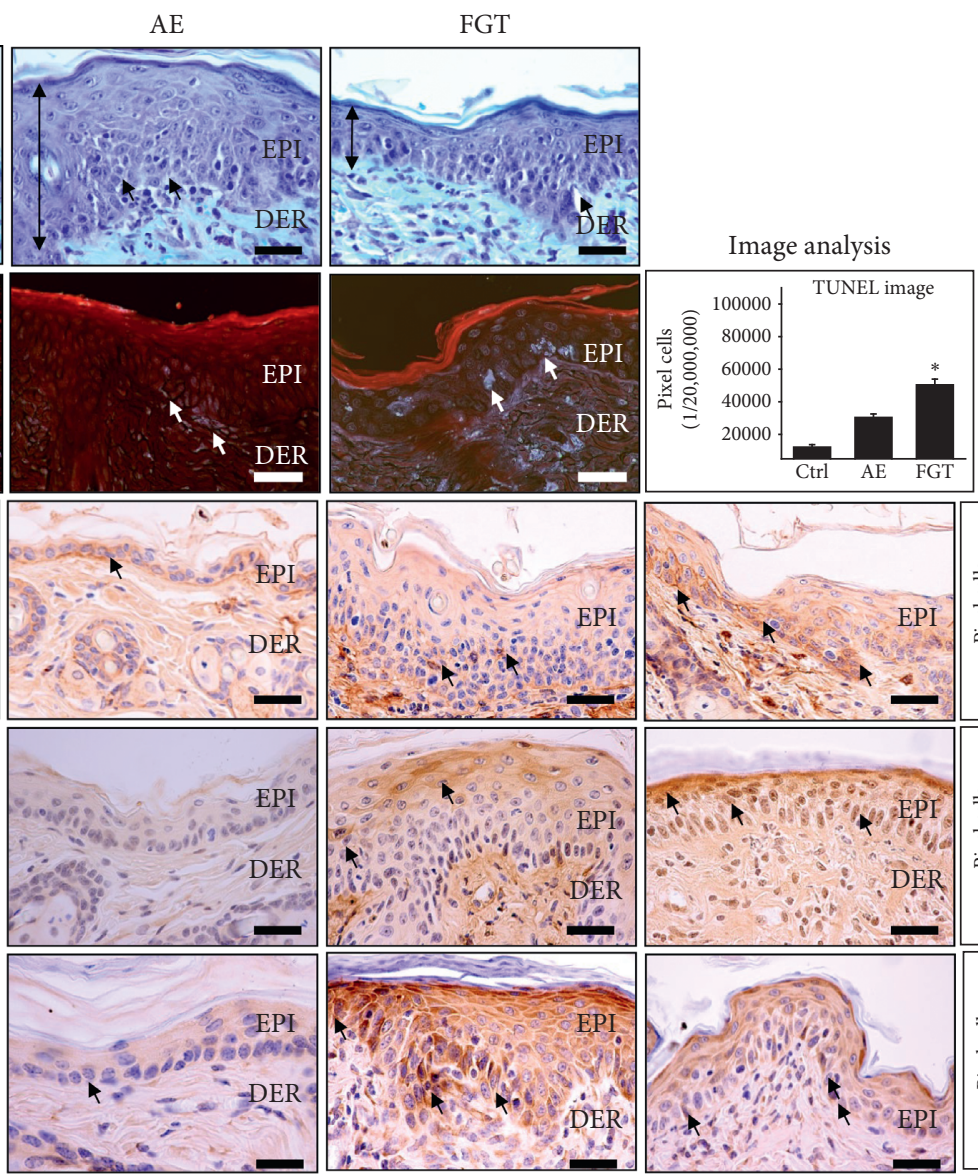

$\mathrm{AE}$

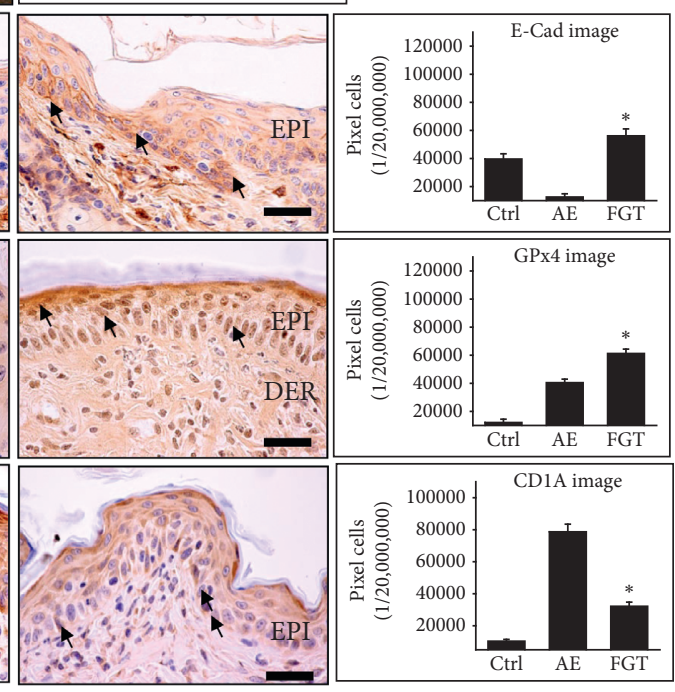

Image analysis

FIGURE 5: The epidermal structure regulatory effects of HTD. HTD improved the epidermal structures in the FGT group. In toluidine blue staining result, epidermal thickness (arrow $(\uparrow)$ indicates), infiltration lymphocytes (arrow indicates dark navy), and intercellular spaces (arrow indicates white) were increased in the AE group but decreased in the FGT group $(p<0.01)$ (toluidine blue staining; bar size, $50 \mu \mathrm{m}$ ). The E-cadherin expression (arrow indicates dark brown) was decreased in the AE group, but significantly increased in the FGT group $(p<0.01)$ (bar size, $50 \mu \mathrm{m})$. The GPx4 expression (arrow indicates dark brown) in the FGT group was remarkably increased compared with the AE group $(p<0.01)$ (bar size, $50 \mu \mathrm{m}$ ). The CD1A expression (arrow indicates dark brown) was significantly increased but decreased in the FGT group $(p<0.01)$. In TUNEL assay, the apoptotic body (arrow indicates white-blue fluorescence) in the FGT group was remarkably increased compared to the AE group (TUNEL assay; bar size, $50 \mu \mathrm{m}$ ). ${ }^{*} p<0.01$, compared with the AE group. Ctrl: normal, AE: AD-induced with no treatment, FGT: AD-induced with HTD treatment, EPI: epidermis, DER: dermis, and E-Cad: E-cadherin.

pixels which were significantly reduced by $57 \%(p<0.01)$ compared with those of the AE group (Figure 6). The results of $\mathrm{p}$-JNK expression were similar to the results for $\mathrm{p}$-ERK. Compared with the levels in the Ctrl group, the p-JNK levels of the AE group were 55,413 $\pm 1355 / 20,000,000$ pixels which were increased by $736 \%$. After HTD, the p-JNK levels decreased. The p-JNK levels in the FGT group were $28,299 \pm 482 / 20,000,000$ pixels which were decreased by $49 \%$ $(p<0.01)$ compared with those of the AE group (Figure 6).

Recently, studies reported that inflammatory cytokines such as tumor necrosis factor- $\alpha$ (TNF- $\alpha$ ), IL- $1 \beta$, and IL-17A induce mTOR activation to promote the epidermal proliferation and reduce the expression of epidermal differentiation markers [49]. Moreover, topical application of the mTOR inhibitor rapamycin to $\mathrm{AD}$ skin lesions resulted in decreased inflammatory cell infiltration and serum IgE levels, suggesting that inhibition of mTOR signaling suppresses $A D$ [50]. Therefore, we performed an immunohistochemical analysis of expression of $\mathrm{p}$-mTOR, the activation form of mTOR, to determine the effects of HTD. The expression of p-mTOR in the AE group was $104,341 \pm 2111 / 20,000,000$ pixels which were significantly increased by $417 \%(p<0.01)$ compared with that of the Ctrl group. In contrast, p-mTOR expression in the FGT group was $66,372 \pm 977 / 20,000,000$ pixels which were significantly reduced by $36 \%(p<0.01)$ compared with that of the $\mathrm{AE}$ group (Figure 6).

Overall, our results indicate that HTD suppresses epidermal inflammation by reducing the infiltration of inflammatory cells and modulating p-ERK, p-JNK, and p-mTOR expression.

3.6. Genistein as an ECS Modulator. Genistein was reported to maintain the homeostasis of the skin barrier by increasing the lipid barrier integrity in NC/Nga mice induced AD [51]. In particular, genistein has been shown to affect ECS by 


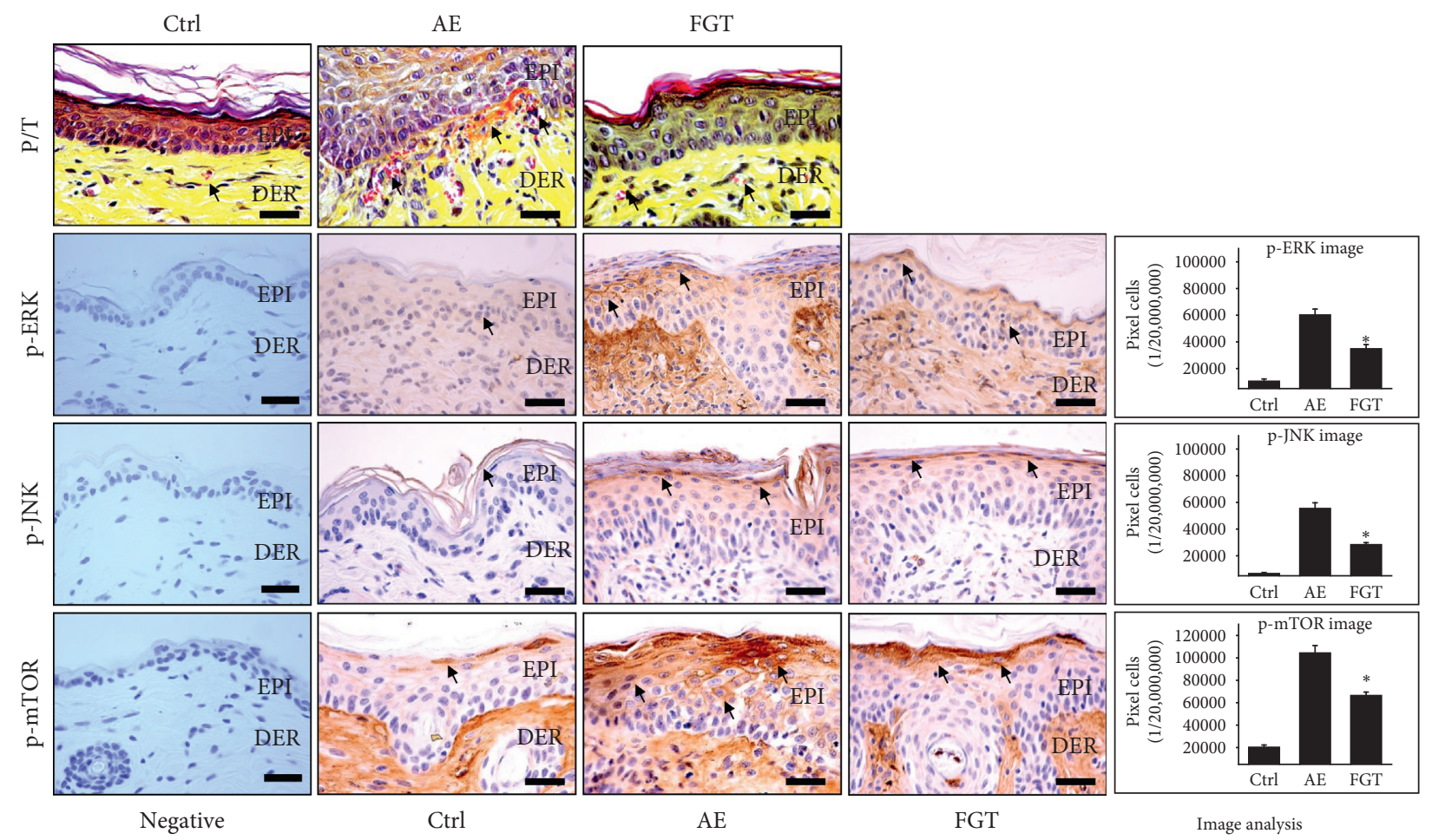

Figure 6: The epidermal inflammation regulatory effects of HTD. HTD reduced the epidermal inflammation in the FGT group. In P/T staining result, infiltration of inflammatory cells and capillary distributions (arrow indicates red) were increased in the AE group but decreased in the FGT group $(p<0.01)(\mathrm{P} / \mathrm{T}$ staining; bar size, $50 \mu \mathrm{m})$. The p-ERK, p-JNK, and p-mTOR expressions (arrow indicates dark brown) were increased in the AE group, but significantly decreased in the FGT group $(p<0.01)$ (bar size, $50 \mu \mathrm{m}) .{ }^{*} p<0.01$ compared with the AE group. Ctrl: normal, AE: AD-induced with no treatment, FGT: AD-induced with HTD treatment, EPI: epidermis, DER: dermis, and P/T: phloxine-tartrazine.

blocking FAAH to inhibit cellular uptake of AEA [52]. In our previous studies, high-performance liquid chromatography (HPLC) analysis of Douchi extract showed that the ratio of genistein was the highest among the active ingredients of Douchi extract [26]. Thus, we confirmed the lipid barrier formation effect of genistein, which acts as an ECS modulator, and compared the effect with PEA, one of the ECS agonists.

To estimate the effect of genistein on the formation of the skin lipid barrier, we performed western blot and measured expression of the levels of involucrin. As a result, treatment with genistein increased the expression level of involucrin in a concentration-dependent manner (Figure 7(b)).

The effects of genistein and PEA were compared by measuring the expression of involucrin using western blot and immunohistochemistry in dermatitis-induced animal models.

Western blotting results showed that the involucrin expression was markedly increased in the GT group. The expression of involucrin in the PTgroup was lower than that in the GT group (Figure 7(d)).

The levels of involucrin the SC were markedly reduced in the DE group, whereas the levels of involucrin in the GT group were increased by $402 \%(p<0.01)$ compared with those in the DE group. Moreover, the expression of involucrin in the PT group was $15,969 \pm 821 / 20,000,000$ pixels which were significantly lower by $27 \%(p<0.01)$ compared to the GT group of $21,743 \pm 717 / 20,000,000$ pixels (Figure 7(e)).

Moreover, these results were consistent with those of TEWL. Genistein significantly moisturized the skin compared to the DE group. The levels of TEWL in the GT group were decreased by $60 \%(p<0.01)$ compared with those of the DE group (Figure $7(\mathrm{c})$ ).

Overall, our results indicate that genistein, one of the active ingredients of Douchi extract, acted as an ECS modulator and showed a better lipid barrier formation effect than PEA.

\section{Discussion}

The epidermis maintains the homeostasis of the skin barrier by constantly repeating the proliferation, differentiation, and cornification of new epidermal cells [53]. Epidermal homeostasis means that keratinocytes undergo differentiation to form a skin barrier called the SC and to continuously maintain the permeability barrier function [54]. Skin diseases such as $\mathrm{AD}$ are caused by abnormalities in the physiological balance required to maintain epidermal homeostasis [55]. In particular, the ECS is intervened in the regulation of skin homeostasis by modulating cell growth, differentiation, and immune and inflammatory responses [13]. Recent evidence revealed that inadequate operation of the ECS can affect skin pathologies, such as skin barrier 
<smiles>O=c1c(-c2ccc(O)cc2)coc2cc(O)cc(O)c12</smiles>

Genistein

(a)

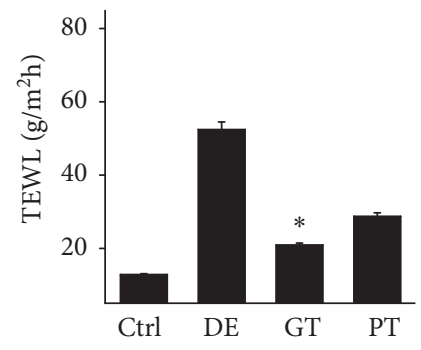

(c)

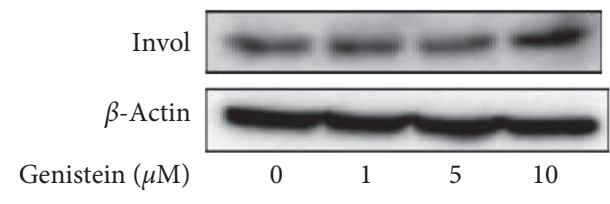

(b)
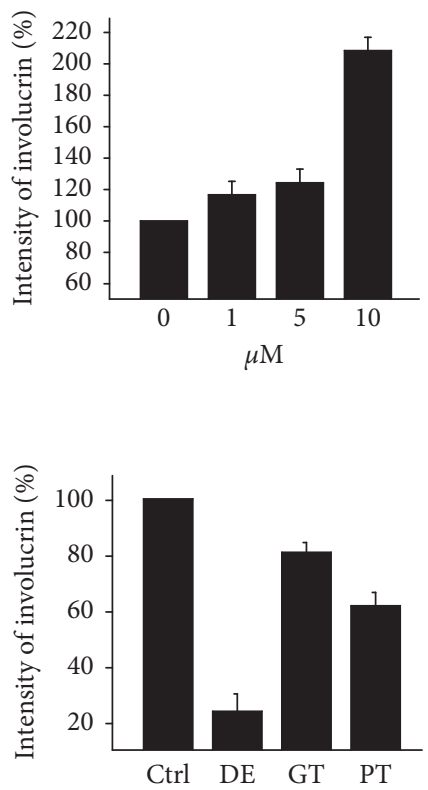

(d)
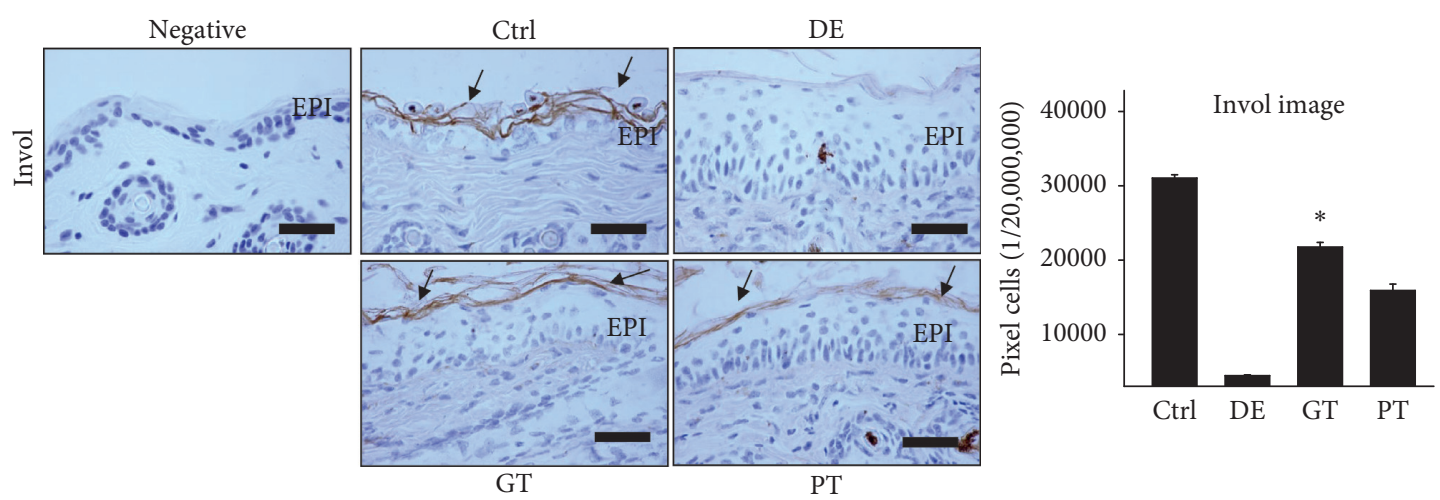

(e)

FIGURE 7: The effect of genistein as ECS modulator. (a) Structure of genistein. (b) Expression of involucrin was detected by western blotting using genistein. $\beta$-Actin protein was used as an internal control. (c) Effect of genistein on TEWL. Genistein significantly moisturized the skin compared to the DE group $(p<0.01)$. (d) In western blotting result, the involucrin expression was markedly increased in the GT group $(p<0.01)$. (e) The expression of involucrin (arrow indicates light brown) was significantly increased in the GT group as compared with the DE group $(p<0.01)$. The expression of involucrin in the PT group was lower than that in the GT group (bar size, $50 \mu \mathrm{m}) .{ }^{*} p<0.01$ compared with the DE group. Ctrl: normal, DE: dermatitis-induced with no treatment, GT: dermatitis-induced with genistein treatment, PT: dermatitis-induced with PEA treatment, EPI: epidermis, Invol: involucrin, and TEWL: transepidermal water loss.

dysfunction in AD [56]. Therefore, the ECS is emerging as a new treatment target for various skin diseases.

The ECS consists of cannabinoid receptors (CBRs), their endogenous ligands, and the enzymes involved in the synthesis and degradation of CBs [57]. Two major CBRs are $\mathrm{CB} 1$ and $\mathrm{CB} 2$, and recently another $\mathrm{G}$ protein-coupled cannabinoid receptor, GPR55, has emerged as a type 3 CBR [58]. Several studies have shown that CB1 agonists relieve inflammatory symptoms [59] by downregulating mast cell activation [60] and decreasing keratinocyte-derived proinflammatory mediators [61]. In addition, CB2 agonists suppress skin inflammation by inhibiting inflammatory cell migration [62], and GPR55, which is found in mast cells, has anti-inflammatory effects by inhibiting mast cell-mediated release of nerve growth factor and reducing angiogenesis
[63]. In our study, the results indicate that HTD generates ECS components such as CB1, CB2, and GPR55. Based on this result, we suggest that HTD alleviates AD symptoms by intervening in the ECS.

As previously mentioned, the keratinocyte differentiation markers, filaggrin, involucrin, and loricrin, have a crucial function by forming the CE to maintain the epidermal barrier [64]. Filaggrin plays an important role in maintaining skin surface hydration by protecting SC integrity and the generation of natural moisturizing factors [65]. Filaggrin expression has been reported to be reduced in $\mathrm{AD}$ [66], and filaggrin mutations have been reported to cause skin inflammation, causing skin barrier damage in $\mathrm{AD}$ and promoting IgE sensitization through the damaged skin barrier [67]. Thus, a filaggrin deficiency vitally related to the 
pathogenesis of $\mathrm{AD}$ [68]. Additionally, the levels of loricrin and involucrin have been reported to be lower in $\mathrm{AD}$ than in healthy skin [69], and the reduced expression of loricrin and involucrin was found to be downregulated by Th2 cytokines through the STAT-6 signaling pathway [36]. In addition, several studies have been conducted on the role of the ECS in the control of epidermal differentiation [70]. Recently, the specific role of $\mathrm{CB} 1$ and $\mathrm{CB} 2$ in keratinocyte differentiation has been reported to have opposite effects in the regulation of epidermal barrier permeability and keratinocyte differentiation [71]. AEA mediates CB1-dependent transcriptional processes related to epidermal differentiation and skin development [14]. According to our results, HTD increased the expression of not only CB1, CB2, and GPR55 but also filaggrin, involucrin, and loricrin. Moreover, HTD improved Lass2 expression and reduced PKC expression. Lass2 is the most widely distributed ceramide synthase in the human body and catalyzes the synthesis of heavy chain ceramides [39]. Ceramides are an important factor in determining the water retention property and epidermal barrier integrity [72]. In $\mathrm{AD}$ skin lesions, reduced ceramide levels were observed [6]. Reduced ceramides affect PKC activation in $\mathrm{AD}$, leading to the production of inflammatory cytokines and barrier disruption [73]. These findings mean that HTD reduced the PKC activation observed in $\mathrm{AD}$ by increasing ceramide synthesis. Based on these results, we suggest that HTD enhances epidermal barrier integrity by promoting keratinocyte proliferation from stratum granulosum (SG) to SC and lipid barrier formation by intervening in ECS.

E-cadherin expression was downregulated on keratinocyte surfaces in the lesional skin of AD [74], and dissolution of the tight junctions that are important for maintaining the barrier function of the epidermis was followed by downregulation of E-cadherin expression [75]. Oxidative stress directly affects epidermal keratinocytes and induces intracellular changes, resulting in edema, spongiosis, and destruction of SC [76]. GPx4 is an antioxidant enzyme that recovers oxidative damage and is increased in expression as a defense mechanism against oxidative stress [77]. Recently, it was revealed that GPx4 regulates the release of apoptotic proteins and plays a critical role in cellular homeostasis through apoptosis [43]. In GPx4 deletion mouse experiments, epidermal hyperplasia, dermal inflammatory infiltration, and reduced keratinocyte adhesion were induced [78]. In our study, HTD maintained the epidermal structure by regulating the expression of E-cadherin and GPx4.

MAPKs, including ERK and JNK, are essential signals for the production of cytokines such as IL-4 and TNF- $\alpha$ [79] and regulate the inflammatory response through nuclear factorkappa B (NF-kB) activation [80]. IL-17, a proinflammatory cytokine, decreases the expression of filaggrin and involucrin in AD via the MAPKs signal pathway [81]. In our study, HTD also modulated the inflammatory response by blocking the MAPK signaling pathway by inhibiting the phosphorylation of ERK and JNK.

In addition, we confirmed the effect of genistein, one of the active ingredients to strengthen our results. In western blotting, genistein increased the expression level of involucrin in a concentration-dependent manner. In comparison with PEA, the expression of involucrin was significantly increased. Thus, the HTD with Douchi extract acts an ECS modulator and showed a better lipid barrier formation effect than PEA.

In this study, we demonstrated the effects of HTD on AD using a $D f E$-induced AD model. HTD improved external pathological features and $\mathrm{AD}$ skin scores and reduced angiogenesis and epithelium hyperplasia. HTD also inhibited IL-4 expression. Furthermore, orally administered Douchi extracts as HTD significantly generated ECS components, such as CB1, CB2, and GPR55, and lipid barrier formation and suppressed hyperplasia and infiltration of inflammatory cells. In particular, the HTD-mediated operation status of ECS was higher than that of the Ctrl group. Therefore, the HTD-mediated alleviation of AD-induced skin lesions was observed in vivo.

In conclusion, $\mathrm{HTD}$ can alleviate symptoms of $\mathrm{AD}$ such as erythema, bleeding, scarring, erosion, and excoriation by maintaining skin homeostasis, improving skin barrier formation, and downregulating inflammation, through ECS intervention. Therefore, this study shows the potential of $\mathrm{HTD}$ for the treatment of $\mathrm{AD}$ as a prophylactic treatment to maintain homeostasis through epidermal structure improvement.

\section{Conclusions}

In this study, we demonstrated that HTD effectively suppressed the development of symptoms of AD using $D f \mathrm{E}$ induced NC/Nga AD mice. These results suggest that HTD intervenes in the ECS to maintain skin homeostasis, thereby maintaining the epidermal structure and downregulating inflammation.

\section{Abbreviations}

HTD: Hataedock

AD: $\quad$ Atopic dermatitis

DfE: $\quad$ Dermatophagoides farinae extract

CB: $\quad$ Cannabinoid receptor type

GPR55: $\quad$ G protein-coupled receptor 55

Lass2: $\quad$ Longevity assurance homolog 2

GPx4: $\quad$ Glutathione peroxidase 4

CD1A: Cluster of differentiation 1a

p-ERK: Phosphorylated extracellular signal-related kinase

p-JNK: $\quad$ Phosphorylated c-Jun amino-terminal kinase

p- Phosphorylated mammalian target of rapamycin

mTOR:

PEA: Palmitoylethanolamide

SC: $\quad$ Stratum corneum

ECS: Endocannabinoid system

CBs: Cannabinoids

AEA: Anandamide

2-AG: 2-Arachidonoylglycerol

FAAH: Fatty acid amide hydrolase

TEWL: Transepidermal water loss

M/T: $\quad$ Masson's trichrome 
P/T: Phloxine-tartrazine

PKC: $\quad$ Protein kinase $\mathrm{C}$

TUNEL: Terminal deoxynucleotidyl transferase dUTP nick end labeling

PVDF: Polyvinylidene difluoride

ANOVA: One-way analysis of variance

CE: $\quad$ Cornified cell envelope

SP: $\quad$ Stratum spinosum

SB: $\quad$ Stratum basale

MAPKs: Mitogen-activated protein kinase

AP-1: $\quad$ Activator protein 1

CBRs: Cannabinoid receptors

CBs: Cannabinoids

SG: $\quad$ Stratum granulosum

IL: Interleukin

TNF- $\alpha$ : Tumor necrosis factor-alpha.

\section{Data Availability}

The data used to support the findings of this study are available from the corresponding author upon request.

\section{Conflicts of Interest}

The authors declare that there are no conflicts of interest.

\section{Authors' Contributions}

Hee-Yeon Kim and Sang-hyun Ahn contributed equally to this work.

\section{Acknowledgments}

This research was supported by the Basic Science Research Program through the National Research Foundation of Korea (NRF) funded by the Ministry of Science and ICT (NRF-2019R1A2C1002443).

\section{References}

[1] W. Peng and N. Novak, "Pathogenesis of atopic dermatitis," Clinical \& Experimental Allergy, vol. 45, no. 3, pp. 566-574, 2015.

[2] J. Spergel and A. S. Paller, "Atopic dermatitis and the atopic march," Journal of Allergy and Clinical Immunology, vol. 112, no. 6, pp. S118-S127, 2003.

[3] K. Kabashima, "New concept of the pathogenesis of atopic dermatitis: interplay among the barrier, allergy, and pruritus as a trinity," Journal of Dermatological Science, vol. 70, no. 1, pp. 3-11, 2013.

[4] P. Elias, L. C. Wood, and K. R. Feingold, "Epidermal pathogenesis of inflammatory dermatoses," American Journal of Contact Dermatitis, vol. 10, no. 3, pp. 119-126, 1999.

[5] H. Y. Lee, "Improvement of skin barrier dysfunction by Scutellaria baicalensis GEOGI extracts through lactic acid fermentation," Journal of Cosmetic Dermatology, vol. 18, no. 1, pp. 183-191, 2019.

[6] G. Imokawa, A. Abe, K. Jin, Y. Higaki, M. Kawashima, and A. Hidano, "Decreased level of ceramides in stratum corneum of atopic dermatitis: an etiologic factor in atopic dry skin?"
Journal of Investigative Dermatology, vol. 96, no. 4, pp. 523526, 1991.

[7] J.-M. Jensen, R. Fölster-Holst, A. Baranowsky et al., "Impaired sphingomyelinase activity and epidermal differentiation in atopic dermatitis," Journal of Investigative Dermatology, vol. 122, no. 6, pp. 1423-1431, 2004.

[8] M. Schafer and S. Werner, "Cancer as an overhealing wound: an old hypothesis revisited," Nature Reviews Molecular Cell Biology, vol. 9, no. 8, pp. 628-638, 2008.

[9] J. Manzanares, M. Julian, and A. Carrascosa, "Role of the cannabinoid system in pain control and therapeutic implications for the management of acute and chronic pain episodes," Current Neuropharmacology, vol. 4, no. 3, pp. 239-257, 2006.

[10] P. Pacher, S. Bátkai, and G. Kunos, "The endocannabinoid system as an emerging target of pharmacotherapy," Pharmacological Reviews, vol. 58, no. 3, pp. 389-462, 2006.

[11] L. K. Miller and L. A. Devi, "The highs and lows of cannabinoid receptor expression in disease: mechanisms and their therapeutic implications," Pharmacological Reviews, vol. 63, no. 3, pp. 461-470, 2011.

[12] T. A. Luger, "Neuromediators-a crucial component of the skin immune system," Journal of Dermatological Science, vol. 30, no. 2, pp. 87-93, 2002.

[13] B. I. Tóth, N. Dobrosi, A. Dajnoki et al., "Endocannabinoids modulate human epidermal keratinocyte proliferation and survival via the sequential engagement of cannabinoid receptor-1 and transient receptor potential vanilloid-1," Journal of Investigative Dermatology, vol. 131, no. 5, pp. 1095-1104, 2011.

[14] M. Maccarrone, M. Di Rienzo, N. Battista et al., "The endocannabinoid system in human keratinocytes," Journal of Biological Chemistry, vol. 278, no. 36, pp. 33896-33903, 2003.

[15] M. Karsak, E. Gaffal, R. Date et al., "Attenuation of allergic contact dermatitis through the endocannabinoid system," Science, vol. 316, no. 5830, pp. 1494-1497, 2007.

[16] M. L. Casanova, C. Blázquez, J. Martínez-Palacio et al., "Inhibition of skin tumor growth and angiogenesis in vivo by activation of cannabinoid receptors," Journal of Clinical Investigation, vol. 111, no. 1, pp. 43-50, 2003.

[17] E. Gaffal, M. Cron, N. Glodde et al., "Cannabinoid 1 receptors in keratinocytes modulate proinflammatory chemokine secretion and attenuate contact allergic inflammation," The Journal of Immunology, vol. 190, no. 10, pp. 4929-4936, 2013.

[18] B. K. Srivastava, R. Soni, J. Z. Patel et al., "Hair growth stimulator property of thienyl substituted pyrazole carboxamide derivatives as a CB1 receptor antagonist with in vivo antiobesity effect," Bioorganic \& Medicinal Chemistry Letters, vol. 19, no. 9, pp. 2546-2550, 2009.

[19] N. Dobrosi, B. I. Tóth, G. Nagy et al., "Endocannabinoids enhance lipid synthesis and apoptosis of human sebocytesviacannabinoid receptor-2-mediated signaling," The FASEB Journal, vol. 22, no. 10, pp. 3685-3695, 2008.

[20] J. M. Walker and A. G. Hohmann, "Cannabinoid mechanisms of pain suppression," Handbook of Experimental Pharmacology, vol. 168, pp. 509-554, Springer, Berlin, Germany, 2005.

[21] M. D. Jhaveri, D. Richardson, and V. Chapman, "Endocannabinoid metabolism and uptake: novel targets for neuropathic and inflammatory pain," British Journal of Pharmacology, vol. 152, no. 5, pp. 624-632, 2007.

[22] K. Tóth, D. A.th, T. Bíró, and A. Oláh, "Cannabinoid signaling in the skin: therapeutic potential of the "C(ut)annabinoid" system," Molecules, vol. 24, no. 5, p. 918, 2019. 
[23] S. Li, Z. Q. Zhang, L. J. Wu, X. G. Zhang, Y. Y. Wang, and Y. D. Li, "Understanding ZHENG in traditional Chinese medicine in the context of neuro-endocrine-immune network," IET Systems Biology, vol. 1, no. 1, pp. 51-60, 2007.

[24] R. A. Muluye, Y. Bian, and P. N. Alemu, "Anti-inflammatory and antimicrobial effects of heat-clearing Chinese herbs: a current review," Journal of Traditional and Complementary Medicine, vol. 4, no. 2, pp. 93-98, 2014.

[25] P. S. Qinghu He and Y. Zhang, TCM Case Studies: External Medicine, People's Medical Publishing House, Beijing, China, 1st edition, 2014.

[26] A. R. Jung, S. H. Ahn, I. S. Park et al., "Douchi (fermented Glycine max Merr.) alleviates atopic dermatitis-like skin lesions in NC/Nga mice by regulation of PKC and IL-4," BMC Complementary and Alternative Medicine, vol. 16, no. 1, p. 416, 2016.

[27] H.-Y. Cha, S.-h. Ahn, J.-H. Cheon, I.-S. Park, J.-T. Kim, and K. Kim, "Hataedock treatment has preventive therapeutic effects in atopic dermatitis-induced NC/nga mice under highfat diet conditions," Evidence-Based Complementary and Alternative Medicine, vol. 2016, Article ID 1739760, 13 pages, 2016.

[28] H.-Y. Kim, S.-H. Ahn, I. Yang, and K. Kim, "Effect of skin lipid barrier formation on Hataedock treatment with Douchi," Journal of Korean Medicine, vol. 38, no. 2, pp. 41-52, 2017.

[29] J. H Hong, S. H. Ahn, J. H. Cheon et al., "Effects of Hataedock with Douchi on 2,4-dinitrofluorobenzene-induced atopic dermatitis-like skin lesion in NC/nga mice," Journal of Physiology \& Pathology in Korean Medicine, vol. 30, no. 2, pp. 109-115, 2016.

[30] J. M. McPartland, G. W. Guy, and V. Di Marzo, "Care and feeding of the endocannabinoid system: a systematic review of potential clinical interventions that upregulate the endocannabinoid system," PloS One, vol. 9, no. 3, Article ID e89566, 2014.

[31] V. Di Marzo, "New approaches and challenges to targeting the endocannabinoid system," Nature Reviews Drug Discovery, vol. 17, no. 9, pp. 623-639, 2018.

[32] A. Dizamatova, K. Zhumanova, G. E. Zhusupova et al., "A new prenylated isoflavonoid from limonium leptophyllum," Natural Product Communications, vol. 14, no. 5, Article ID 1934578X19844137, 2019.

[33] L. Thors, J. Burston, B. Alter et al., "Biochanin A, a naturally occurring inhibitor of fatty acid amide hydrolase," British Journal of Pharmacology, vol. 160, no. 3, pp. 549-560, 2010.

[34] D. Y. M. Leung, R. L. Hirsch, L. Schneider et al., "Thymopentin therapy reduces the clinical severity of atopic dermatitis," Journal of Allergy and Clinical Immunology, vol. 85, no. 5, pp. 927-933, 1990.

[35] W. J. Lee, K. H. Park, H. W. Cha et al., "The expression of involucrin, loricrin, and filaggrin in cultured sebocytes," Annals of Dermatology, vol. 26, no. 1, pp. 134-137, 2014.

[36] B. E. Kim, D. Y. M. Leung, M. Boguniewicz, and M. D. Howell, "Loricrin and involucrin expression is downregulated by Th2 cytokines through STAT-6," Clinical Immunology, vol. 126, no. 3, pp. 332-337, 2008.

[37] A. Taïeb, "Hypothesis: from epidermal barrier dysfunction to atopic disorders," Contact Dermatitis, vol. 41, no. 4, pp. 177-180, 1999.

[38] N. A. Bourbon, L. Sandirasegarane, and M. Kester, "Ceramide-induced inhibition of akt is mediated through protein kinase c $\zeta$," Journal of Biological Chemistry, vol. 277, no. 5, pp. 3286-3292, 2002.
[39] E. L. Laviad, L. Albee, I. Pankova-Kholmyansky et al., "Characterization of ceramide synthase 2," Journal of Biological Chemistry, vol. 283, no. 9, pp. 5677-5684, 2008.

[40] T. Bieber, “Atopic dermatitis," Annals of Dermatology, vol. 22, no. 2, pp. 125-137, 2008.

[41] K. S. Stenn, A. K. Balin, T. Higgins, and J. O. Stenn, "Spongiosis," Journal of the American Academy of Dermatology, vol. 5, no. 2, pp. 213-214, 1981.

[42] S. Blunder, S. Kõks, G. Kõks et al., "Enhanced expression of genes related to xenobiotic metabolism in the skin of patients with atopic dermatitis but not with ichthyosis vulgaris," Journal of Investigative Dermatology, vol. 138, no. 1, pp. 98108, 2018.

[43] H. Liang, Q. Ran, Y. C. Jang et al., "Glutathione peroxidase 4 differentially regulates the release of apoptogenic proteins from mitochondria," Free Radical Biology and Medicine, vol. 47, no. 3, pp. 312-320, 2009.

[44] N. Novak, T. Bieber, and S. Kraft, "Immunoglobulin e-bearing antigen-presenting cells in atopic dermatitis," Current Allergy and Asthma Reports, vol. 4, no. 4, pp. 263-269, 2004.

[45] V. Oji, N. Seller, A. Sandilands et al., "Ichthyosis vulgaris: novelFLGmutations in the German population and high presence of CD1a+ cells in the epidermis of the atopic subgroup," British Journal of Dermatology, vol. 160, no. 4, pp. 771-781, 2009.

[46] A. De Benedetto, A. Kubo, and L. A. Beck, "Skin barrier disruption: a requirement for allergen sensitization?" Journal of Investigative Dermatology, vol. 132, no. 3, pp. 949-963, 2012.

[47] Y. L. Zhang and C. Dong, "MAP kinases in immune responses," Cellular \&amp; Molecular Immunology, vol. 2, no. 1, pp. 20-27, 2005.

[48] J. Zhong and J. M. Kyriakis, "Dissection of a signaling pathway by which pathogen-associated molecular patterns recruit the JNK and p38 MAPKs and trigger cytokine release," Journal of Biological Chemistry, vol. 282, no. 33, pp. 2424624254, 2007.

[49] C. Buerger, N. Shirsath, V. Lang et al., "Inflammation dependent mTORC1 signaling interferes with the switch from keratinocyte proliferation to differentiation," PLoS One, vol. 12, no. 7, Article ID e0180853, 2017.

[50] F. Yang, M. Tanaka, M. Wataya-Kaneda et al., "Topical application of rapamycin ointment amelioratesDermatophagoides farinabody extract-induced atopic dermatitis in NC/Nga mice," Experimental Dermatology, vol. 23, no. 8, pp. 568-572, 2014.

[51] T. Sakai, M. Kogiso, K. Mitsuya, T. Komatsu, and S. Yamamoto, "Genistein suppresses development of spontaneous atopic-like dermatitis in NC/Nga mice," Journal of Nutritional Science and Vitaminology, vol. 52, no. 4, pp. 293-296, 2006.

[52] L. Thors, J. Eriksson, and C. J. Fowler, "Inhibition of the cellular uptake of anandamide by genistein and its analogue daidzein in cells with different levels of fatty acid amide hydrolase-driven uptake," British Journal of Pharmacology, vol. 152, no. 5, pp. 744-750, 2007.

[53] K. Natsuga, "Epidermal barriers," Cold Spring Harbor Perspectives in Medicine, vol. 4, no. 4, Article ID a018218, 2014.

[54] C. Blanpain and E. Fuchs, "Epidermal homeostasis: a balancing act of stem cells in the skin," Nature Reviews Molecular Cell Biology, vol. 10, no. 3, pp. 207-217, 2009.

[55] J.-K. Kim, J.-H. Lee, I.-H. Bae, D.-B. Seo, and S.-J. Lee, "Beneficial effect of a collagen peptide supplement on the epidermal skin barrier," Korean Journal of Food Science and Technology, vol. 43, no. 4, pp. 458-463, 2011. 
[56] P. Kupczyk, A. Reich, and J. C. Szepietowski, "Cannabinoid system in the skin - a possible target for future therapies in dermatology," Experimental Dermatology, vol. 18, no. 8, pp. 669-679, 2009.

[57] M. Salzet, "Invertebrate molecular neuroimmune processes," Brain Research Reviews, vol. 34, no. 1-2, pp. 69-79, 2000.

[58] H. Yang, J. Zhou, and C. Lehmann, "GPR55-a putative "type 3" cannabinoid receptor in inflammation," Journal of Basic and Clinical Physiology and Pharmacology, vol. 27, no. 3, pp. 297-302, 2016.

[59] H. J. Kim, B. Kim, B. M. Park et al., "Topical cannabinoid receptor 1 agonist attenuates the cutaneous inflammatory responses in oxazolone-induced atopic dermatitis model," International Journal of Dermatology, vol. 54, no. 10, pp. e401-e408, 2015.

[60] G. Nam, S. K. Jeong, B. M. Park et al., "Selective cannabinoid receptor-1 agonists regulate mast cell activation in an oxazolone-induced atopic dermatitis model," Annals of Dermatology, vol. 28, no. 1, pp. 22-29, 2016.

[61] E. Gaffal, M. Cron, N. Glodde, and T. Tüting, "Anti-inflammatory activity of topical THC in DNFB-mediated mouse allergic contact dermatitis independent of $\mathrm{CB} 1$ and $\mathrm{CB} 2$ receptors," Allergy, vol. 68, no. 8, pp. 994-1000, 2013.

[62] T. Haruna, M. Soga, Y. Morioka et al., "The inhibitory effect of S-777469, a cannabinoid type 2 receptor agonist, on skin inflammation in mice," Pharmacology, vol. 99, no. 5-6, pp. 259-267, 2017.

[63] G. Cantarella, M. Scollo, L. Lempereur, G. Saccani-Jotti, F. Basile, and R. Bernardini, "Endocannabinoids inhibit release of nerve growth factor by inflammation-activated mast cells," Biochemical Pharmacology, vol. 82, no. 4, pp. 380-388, 2011.

[64] M. Noh, H. Yeo, J. Ko, H. K. Kim, and C.-H. Lee, "MAP17 is associated with the T-helper cell cytokine-induced downregulation of filaggrin transcription in human keratinocytes," Experimental Dermatology, vol. 19, no. 4, pp. 355-362, 2010.

[65] J. Levin, S. F. Friedlander, and J. Q. Del Rosso, "Atopic dermatitis and the stratum corneum: Part 1: the role of filaggrin in the stratum corneum barrier and atopic skin," The Journal of Clinical and Aesthetic Dermatology, vol. 6, no. 10, pp. 16-22, 2013.

[66] T. Seguchi, C. Chang-Yi, S. Kusuda, M. Takahashi, K. Aisu, and T. Tezuka, "Decreased expression of filaggrin in atopic skin," Archives of Dermatological Research, vol. 288, no. 8, pp. 442-446, 1996.

[67] G. M. O’Regan and A. D. Irvine, “The role of filaggrin in the atopic diathesis," Clinical \& Experimental Allergy, vol. 40, no. 7, pp. 965-972, 2010.

[68] B. Cabanillas and N. Novak, "Atopic dermatitis and filaggrin," Current Opinion in Immunology, vol. 42, pp. 1-8, 2016.

[69] J. Jarzab, B. Filipowska, J. Zebracka et al., "Locus 1q21 gene expression changes in atopic dermatitis skin lesions: deregulation of small proline-rich region 1A," International Archives of Allergy and Immunology, vol. 151, no. 1, pp. 28-37, 2010.

[70] M. Pucci, V. Pirazzi, N. Pasquariello, and M. Maccarrone, "Endocannabinoid signaling and epidermal differentiation," European Journal of Dermatology, vol. 21, no. S1, pp. 29-34, 2011.

[71] T. Roelandt, C. Heughebaert, S. Bredif et al., "Cannabinoid receptors 1 and 2 oppositely regulate epidermal permeability barrier status and differentiation," Experimental Dermatology, vol. 21, no. 9, pp. 688-693, 2012.
[72] J. Hara, K. Higuchi, R. Okamoto, M. Kawashima, and G. Imokawa, "High-expression of sphingomyelin deacylase is an important determinant of ceramide deficiency leading to barrier disruption in atopic Dermatitis1," Journal of Investigative Dermatology, vol. 115, no. 3, pp. 406-413, 2000.

[73] S. Pastore, F. Mascia, and G. Girolomoni, "The contribution of keratinocytes to the pathogenesis of atopic dermatitis," European Journal of Dermatology, vol. 16, no. 2, pp. 125-131, 2006.

[74] A. Trautmann, F. Altznauer, M. Akdis et al., "The differential fate of cadherins during T-cell-induced keratinocyte apoptosis leads to spongiosis in eczematous dermatitis," Journal of Investigative Dermatology, vol. 117, no. 4, pp. 927-934, 2001.

[75] J. P. Thiery and J. P. Sleeman, "Complex networks orchestrate epithelial-mesenchymal transitions," Nature Reviews Molecular Cell Biology, vol. 7, no. 2, pp. 131-142, 2006.

[76] H. Ji and X.-K. Li, "Oxidative stress in atopic dermatitis," Oxidative Medicine and Cellular Longevity, vol. 2016, Article ID 2721469, 8 pages, 2016.

[77] H. Liang, H. V. Remmen, V. Frohlich, J. Lechleiter, A. Richardson, and Q. Ran, "Gpx4 protects mitochondrial ATP generation against oxidative damage," Biochemical and Biophysical Research Communications, vol. 356, no. 4, pp. 893-898, 2007.

[78] A. Sengupta, U. F. Lichti, B. A. Carlson et al., "Targeted disruption of glutathione peroxidase 4 in mouse skin epithelial cells impairs postnatal hair follicle morphogenesis that is partially rescued through inhibition of COX-2," Journal of Investigative Dermatology, vol. 133, no. 7, pp. 1731-1741, 2013.

[79] P. F.-Y. Cheung, C.-K. Wong, A. W.-Y. Ho, S. Hu, D.-P. Chen, and C. W.-K. Lam, "Activation of human eosinophils and epidermal keratinocytes by Th2 cytokine IL-31: implication for the immunopathogenesis of atopic dermatitis," International Immunology, vol. 22, no. 6, pp. 453-467, 2010.

[80] S. Arbabi and R. V. Maier, "Mitogen-activated protein kinases," Critical Care Medicine, vol. 30, no. 1 Suppl, pp. s74-49, 2002.

[81] Q. Tan, H. Yang, E. Liu, and H. Wang, "P38/ERK MAPK signaling pathways are involved in the regulation of filaggrin and involucrin by IL-17," Molecular Medicine Reports, vol. 16, no. 6, pp. 8863-8867, 2017. 